\title{
Influence of two different cement space settings and three different cement types on the fit of polymer-infiltrated ceramic network material crowns manufactured using a complete digital workflow
}

\author{
Rinet Dauti ${ }^{1} \cdot$ Bledar Lilaj $^{1} \cdot$ Patrick Heimel $^{2} \cdot$ Andreas Moritz $^{1} \cdot$ Andreas Schedle $^{1}$ (D) Barbara Cvikl $^{1,3}$
}

Received: 25 April 2019 / Accepted: 5 August 2019/Published online: 13 September 2019

(C) The Author(s) 2019

\begin{abstract}
Objectives The study evaluates the influence of two spacer settings and three resin luting materials on the marginal and internal fit of polymer-infiltrated ceramic network (PICN) material crowns manufactured using a complete digital workflow.

Methods Optical impressions of fifty identical dies were performed using the 3M scanner (software version 5.0.2). Twenty crowns were designed using Ceramill Mind (version 3.4.10.1163), from which ten with spacer setting of $50 \mu \mathrm{m}(\mathrm{G} 1)$ and ten with $80 \mu \mathrm{m}(\mathrm{G} 2)$. Thirty crowns (spacer setting of $50 \mu \mathrm{m}$ ) were divided into three groups corresponding to the resin materials used as follows: RelyX Unicem (RX), Variolink Esthetic (VLE), and Nexus 3 (NX3). All crowns were milled from Vita Enamic blocks. After micro-CT scanning, absolute marginal discrepancy (AMD), internal gap (IG), total cement space volume (TCV), and marginal porosities (VP) were measured.

Results Significant difference was detected on the VP between the RX and NX3 group $(p=0.033)$. The mean values of all parameters were the following: AMD ( $\mu \mathrm{m})$ : G1 182.6, G2 253.7, RX 210.8, VLE 195.5, NX3 186.6; IG ( $\mu \mathrm{m})$ : G1 215.6, G2 173.1, RX 171.1, VLE 198.6, NX3 203; TCV (mm³): G1 22.9, G2 20.49, RX 17.57, VLE 17.49, NX3 20.59; VP (mm³): G1 0.26, G2 0.34, RX 0.32, VLE 0.46, NX3 0.54.

Conclusions Fit of PICN material crowns was not significantly influenced by increasing the spacer settings and cementation with different resin materials. Additionally, RelyX Unicem showed significantly less porosities as compared with Nexus3.

Clinical relevance Both $50 \mu \mathrm{m}$ and $80 \mu \mathrm{m}$ virtual spacer settings can be suggested for the manufacture of PICN crowns when Ceramill Mind (version 3.4.10.1163) is used. Furthermore, a self-adhesive system can be recommended for the cementation.
\end{abstract}

Keywords Cementspace parameters $\cdot$ Accuracy offit $\cdot$ Cement porosities $\cdot$ Digital workflow $\cdot$ Polymer-infiltrated ceramic network material $\cdot$ Micro-CT

Andreas Schedle

andreas.schedle@meduniwien.ac.at

Rinet Dauti

rinet.dauti@meduniwien.ac.at

Bledar Lilaj

bledar.lilaj@meduniwien.ac.at

Patrick Heimel

patrick.heimel@gmail.com

Andreas Moritz

andreas.moritz@meduniwien.ac.at
Barbara Cvikl

barbara.cvik1@meduniwien.ac.at

1

Department of Conservative Dentistry and Periodontology, University Clinic of Dentistry, Medical University of Vienna, Sensengasse 2A, 1090 Vienna, Austria

2 Karl Donath Laboratory for Hard Tissue and Biomaterial Research, University Clinic of Dentistry, Medical University of Vienna, Sensengasse 2A, 1090 Vienna, Austria

3 Department of Conservative Dentistry, Sigmund Freud University, Vienna, Austria 


\section{Introduction}

CAD/CAM (computer-aided design/computer-aided manufacturing) technologies strongly enhanced the development of highly esthetic materials for the chair-side use in restorative dentistry [1]. Vita Enamic (Vita Zahnfabrik, Bad Säckingen, Germany), for example, is a representative material consisting of a fine glass ceramic three-dimensional network which is infiltrated with a mixture of urethane dimethacrylate (UDMA) and triethylene glycol dimethacrylate (TEGDMA) monomers [2,3]. This composition provides ceramic-like characteristics such as fine surface structure and good esthetic properties. Furthermore, the reduced brittleness and higher fracture toughness is favorable against loading forces and enhances the milling procedure in the manufacturing process of the restoration [4]. In addition, the polymer structure enables the generation of smoother and thinner margins with fewer irregularities which on one hand support the preservation of tooth structure and on the other hand enhance the accuracy in the marginal area of the restoration [5].

Adequate accuracy of fit is crucial for the long-term success of dental restorations [6]. Holmes et al. established measuring parameters to define the accuracy of fit and moreover to compare the results of various studies. The absolute marginal discrepancy (AMD) represents the distance that reflects the largest error in the marginal area of a restoration and the internal gap (IG) reflects the errors in the internal cement space [7]. Different reference values for AMD and IG have been defined as clinically acceptable; however, most of the authors agree that marginal gaps below $120 \mu \mathrm{m}$ are suitable for an adequate fit [8]. An evenly distributed cement layer with low IG values is important for the correct seating and ensures better mechanical properties [6]. Various methods have been described regarding the evaluation of fit of restorations using either a destructive or a non-destructive approach [9]. The evaluation with micro-CT, a non-destructive method, allows not only 2D measurements of distances like AMD and IG but also the quantification of the cement space volume and the volume of porosities inside the luting agent. Consequently, the accuracy of the final restoration can be assessed after cementation as well as in-between working steps [10].

Every working step conducted during the clinical procedure or throughout the $\mathrm{CAD} / \mathrm{CAM}$ manufacturing process influences the accuracy of fit of the restoration. Besides the preparation design, the impression technique, and the milling procedure, the setting of the cement space in the design software and the cementation procedure with the luting agent itself play a crucial role $[6,11,12]$. A certain width of the cement space provides space for the luting agent and facilitates its distribution in the axial area [13]. The width of the cement space affects both the internal gap dimension and the marginal gap. However, to our knowledge, regarding polymer-infiltrated ceramic network (PICN) material crowns, exact recommendations for spacer settings in the design software are still missing. Iwai et al., for example, concluded that zirconia copings with a cement space set to $60 \mu \mathrm{m}$ showed better marginal adaptation than copings with cement space set to 10 and $30 \mu \mathrm{m}$ [14]. Similarly, Kale et al. reported that cement space settings greater than $30 \mu \mathrm{m}$ improve the marginal fit of monolithic zirconia crowns [11], while Yildirim et al. found clinically acceptable marginal and internal adaptation values for PICN material crowns using spacer settings of $40 \mu \mathrm{m}$ [15]. Furthermore, according to Shim et al., spacer settings of $80 \mu \mathrm{m}$ showed better repeatability of marginal and internal fit values [16]. Due to these discrepancies, one aim of this study was to compare the influence of two different spacer settings (50 $\mu \mathrm{m}$ and $80 \mu \mathrm{m})$ on the marginal and internal gap, the cement volume, and the volume of porosities in the marginal area of PICN material crowns.

Besides the spacer setting, the choice of a convenient luting agent and the cementation procedure itself influence the accurate seating and the final marginal and internal discrepancies of the restoration [12]. Furthermore, in order to achieve a better marginal seal and prevent an adhesion failure, proper pre-treatments of the inner surface of PICN material restorations are necessary. According to Peumans et al. Vita Enamic blocks show better adhesion results when a pre-treatment with hydrofluoric acid and silane is performed [17]. The selection of the luting agent is based on various factors such as the type of restoration, the material properties, the preparation design, and the physical and chemical characteristics of the material [18]. However, despite an accurate choice of the luting agent and correct seating, the formation of porosities in the cement space during the cementation procedure could negatively influence the long-term success of the restoration. Factors like the mixing method of the luting agent or the particle size might amplify the formation of porosities [19]. Considering the importance of the right selection of the luting agent, the second aim of the study was to evaluate the influence of three different resin luting materials on the marginal and the internal gap of PICN material crowns. Furthermore, the cement space volume and the volume of porosities in the marginal area of PICN material crowns were investigated.

\section{Materials and methods}

\section{Preparation}

Preparation of a right mandibular typodont tooth (KaVo Dental, Biberach, Germany) was conducted for a crown restoration with a PICN material (Vita Enamic). The circumferential reduction was $0.8-1.2 \mathrm{~mm}$ with a convergence angle of $6^{\circ}$ including a chamfer finish line of $0.8 \mathrm{~mm}$. The occlusal reduction amounted $1.5 \mathrm{~mm}$. The master die was then 
replicated 50 times using a high-quality vinyl silicone (DUOSIL D, SHERA Werkstoff-Technologie, Lemförde, Germany) and a highly precise model resin (Mirapont, Hager \& Werken, Duisburg, Germany). For the first aim of the study, 20 dies were randomly chosen and divided into two groups of ten each, whereas for the second aim, the remaining 30 dies were divided into three groups (Fig. 1).

All dies were marked with a number and fixed in a silicone holder for the impression taking procedure.

\section{Digital impression and crown fabrication}

The dies were digitized using the $3 \mathrm{M}$ TDS intra-oral scanner (3M ESPE, Seefeld, Germany, software version 5.0.2). Before the scanning process, the dies were sprayed with a titanium dioxide-based sprayer (3M High-Resolution Sprayer, 3M ESPE) according to the manufacturer's protocol and digitized starting with the occlusal surface then continuing to the lingual and buccal surface. Afterwards, the data were uploaded to the $3 \mathrm{M}$ servers and made available for download. Subsequently, the 3D data were imported into the design software (Ceramill Mind, Amman Girrbach GmbH, Pforzheim, Germany, version 3.4.10.1163) for the virtual design of the PICN material crowns. All designing and manufacturing steps were conducted by an experienced dental technician.

For the first aim of the study, the crowns of the first group were designed with a spacer setting of $50 \mu \mathrm{m}$ for the internal gap and $0 \mu \mathrm{m}$ for the marginal gap. The crowns of the second group were designed with an internal gap of $80 \mu \mathrm{m}$ and marginal gap of $0 \mu \mathrm{m}$.

For the second aim, the crowns of all three groups were designed with the same fitting parameters - an internal gap of $50 \mu \mathrm{m}$ and marginal gap of $0 \mu \mathrm{m}$.

All crowns were milled from PICN material blocks (Vita Enamic) in a 5 axis milling machine (Ceramill Motion 2 (5×),
Amman Girrbach GmbH, software version 3.4.8). Prior to cementation, the crowns were placed on their respective dies and checked for irregularities using a microscope at $\times 10$ magnification (Stemi DV4 Spot, Carl Zeiss Microscopy, Jena, Germany).

\section{Cementation procedure}

For the first aim, the crowns of both groups were cemented using self-adhesive universal resin material (RelyX Unicem Aplicap, 3M ESPE). A hydrofluoric acid gel (VITA CERAMICS ETCH (5\%), Vita Zahnfabrik $\mathrm{GmbH}$ ) for etching was applied for $60 \mathrm{~s}$ in the inner surface of the crowns and rinsed with water for $60 \mathrm{~s}$. Subsequently, the surface was silanated (Monobond Plus, Ivoclar Vivadent AG, Schaan, Liechtenstein). Afterwards, the resin material was mixed for $10 \mathrm{~s}$ in a capsule mixing unit (RotoMix, 3M ESPE) and applied in the crowns, which had been seated on their respective dies using finger pressure for 2-3 s. The specimens were placed instantly in a seating pressure device for $5 \mathrm{~min}$, where a standardized pressure of $20 \mathrm{~g} / \mathrm{mm}^{2}$ through a plunger was exerted as described by Goracci et al. [20]. At the same time, residual resin material was removed using foam pellets (Pele Tim, Voco, Cuxhaven, Germany), and subsequently, a light curing of the material was performed on each surface of the crown (mesial, distal, buccal, and lingual) for $20 \mathrm{~s}$ using a curing lamp (Bluephase 20i, Ivoclar Vivadent AG) with an intensity of $1200 \mathrm{~mW} / \mathrm{cm}^{2}$.

For the second aim, the crowns of the three groups were pretreated in the same manner as described above. The crowns of the first group (RX) were cemented using RelyX Unicem Aplicap. The crowns of the second group (VLE) were cemented using a dual-curing resin-based material (Variolink Esthetic DC, Ivoclar Vivadent). Prior to cementation, the dies were treated with a self-etching primer (AdheSE Primer,
Fig. 1 Study workflow showing the manufacturing, the cementation, and the measuring process

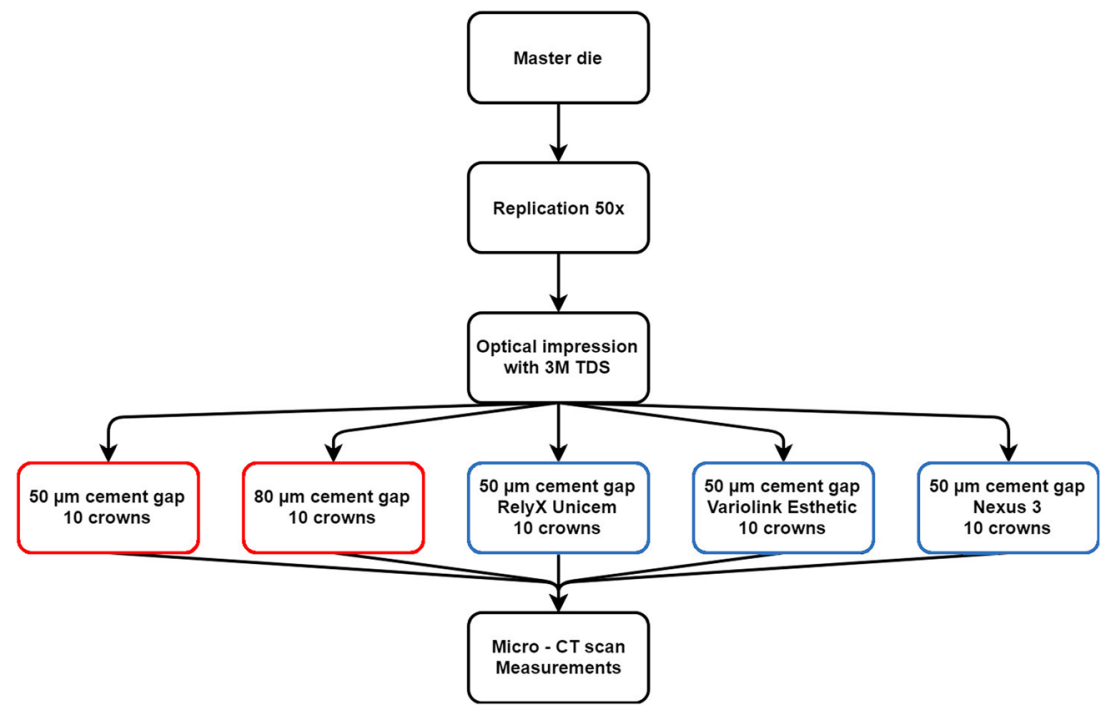


Ivoclar Vivadent) for $30 \mathrm{~s}$ and then dried with air. An adhesive (AdheSE bonding agent, Ivoclar Vivadent) was applied on the prepared surface, scrubbing it for $20 \mathrm{~s}$ and then light-cured for $10 \mathrm{~s}$ using the Bluephase $20 \mathrm{i}$ at an intensity of $1200 \mathrm{~mW} / \mathrm{cm}^{2}$. In the internal surface of the crown, Variolink Esthetic DC cement was applied with an automix syringe, and the crowns were seated on their respective dies in the same way as described above. Glycerine gel was applied to the margins after residual resin material removal in order to prevent oxygen inhibition of polymerization. The third group (NX3) of the crowns was cemented also using resin-based material (Nexus 3, Kerr Corporation, Orange, USA). The working steps for the pre-treatment, seating, and curing were the same as in the previous group with the difference that OptiBond XTR (Kerr Corporation) was used as a primer by scrubbing it for $15 \mathrm{~s}$ and by thin drying with air for $5 \mathrm{~s}$. The adhesive (OptiBond XTR, Kerr Corporation) was applied and then dried for $15 \mathrm{~s}$ respectively. All used materials with their composition and the manufacturers are listed in Table 1.

After the cementation process, all specimens were cleaned with alcohol $70 \%$ vol. and checked for irregularities under microscope prior to micro-CT scan (Fig. 2).

\section{Micro-CT scan and measurements}

A 3D inspection device with a semi-automatic X-ray (Viscom X8060, Viscom AG, Hannover, Germany) was used to perform the scans of the cemented crowns. The device was operated at $130 \mathrm{kV}$ with tube current of $160 \mu \mathrm{A}$ using a $5-\mathrm{mm} \mathrm{Cu}$ filter. Calibration and image correction were performed before every scan procedure in order to achieve the best possible contrast. For each specimen, 1440 images were carried out with a resolution of $9.77 \mu \mathrm{m}$ per pixel. The reconstruction of the data was performed using reconstruction software (XVRCT, Viscom AG, software version 1.07) with a resolution of $10 \mu \mathrm{m}$ per voxel. The reconstructed files (CTR) were converted into tagged image file format (TIFF) for the subsequent segmentation and measuring procedure.

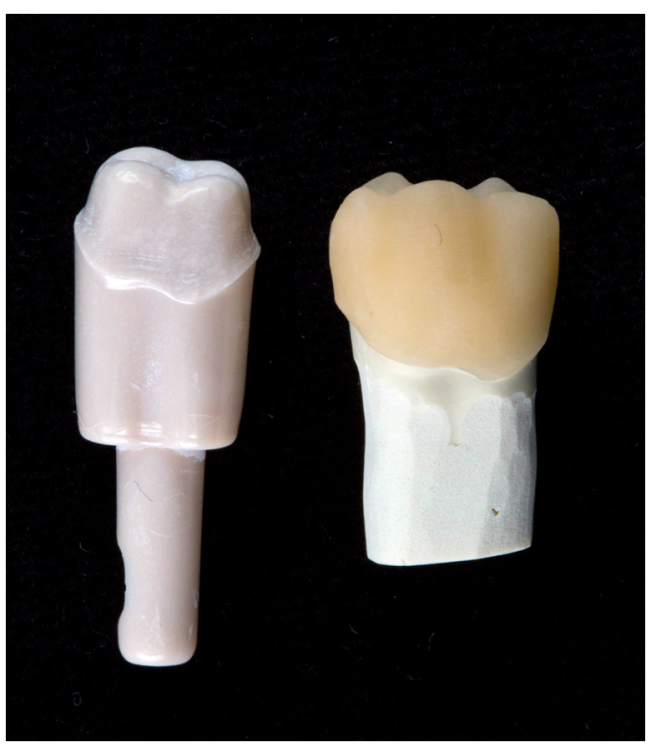

Fig. 2 Photo of the master die and the cemented PICN material crown

The segmentation of the reconstructed files was performed using segmentation software (Definiens Developer XD 2.1.1, Definiens AG, Munich, Germany). In the first step, a rough pre-segmentation was performed in order to achieve a relative separation of the background, the die, the crown, and the cement space.

In the further steps, the objects were optimized by several different intensity thresholds and surface tension constraints to ensure that the gap was not bridged where it was very narrow. Finally, a region of $200 \mu \mathrm{m}$ around the cement space was improved, and the threshold was determined to detect the porosities inside the cement layer (letter D in Fig. 3) and the porosities in contact with the external environment, as shown with letter B in Fig. 3. Volumetric measurements of the total cement space (TCV) and porosities in contact with the external environment (VP) were directly performed with Definiens. The absolute marginal discrepancy (AMD), which represents the distance between the preparation margin and the crown margin (distance A in Fig. 3) as defined by Holmes et al. [7], was measured in 150 equidistant points circumferentially

Table 1 Materials used in the study, manufacturers, and composition

\begin{tabular}{|c|c|c|}
\hline Materials & Manufacturer & Composition \\
\hline AdheSE Primer & Ivoclar Vivadent & Phosphonic acid acrylate, bis-acrylamide derivative \\
\hline AdheSE Adhesive & Ivoclar Vivadent & Dimethacrylates, hydroxyethyl methacrylate, highly dispersed silicon dioxide \\
\hline Monobond Plus & Ivoclar Vivadent & Alcohol solution of silane methacrylate, phosphoric acid methacrylate, and sulfide methacrylate \\
\hline Nexus 3 & Kerr Corporation & TEGDMA, bis-GMA, fluoroaluminosilicate glass, activators, stabilizers, radiopaque agent \\
\hline Optibond XTR Primer & Kerr Corporation & GPDM, hydrophilic co-monomers, water, ethanol, acetone \\
\hline Optibond XTR Bond & Kerr Corporation & Resin monomers, HEMA, inorganic fillers, ethanol \\
\hline RelyX Unicem Aplicap & 3M ESPE & $\begin{array}{l}\text { Alkaline fillers, silanated fillers initiator, pigments, methacrylate monomers containing } \\
\text { phosphoric acid groups, methacrylate monomers }\end{array}$ \\
\hline Variolink Esthetic DC & Ivoclar Vivadent & $\begin{array}{l}\text { UMDA and further methacrylate monomers, ytterbium trifluoride, spheroid mixed oxide, } \\
\text { initiators, stabilizers, and pigments }\end{array}$ \\
\hline VITA CERAMICS ETCH 5\% & Vita Zahnfabrik & Sulfuric acid, hydrofluoric acid, ethanol \\
\hline
\end{tabular}


using Image J. The internal gap, which is the distance between the crown inner surface and the surface of the die (distance $\mathrm{C}$ in Fig. 3), was measured with the BoneJ Plugin for Image $\mathrm{J}$ using the Tb.Th (trabecular thickness) algorithm [21-23]. The cement space thickness and the porosities of representative specimens of each group are shown in Fig. 4.

Representative micro-CT images with visualization of porosities in the marginal area with contact to the external environment are displayed in Fig. 5. More details on the segmentation and the measuring process are available in a previous publication of the authors [24].

\section{Statistical analysis}

The statistical analysis was performed using SPSS Statistics (IBM Corporation, Armonk, USA, version 21) at a significance level of $95 \%$. Mean value, minimum, maximum, and standard deviation for the measuring parameters were calculated. The data are represented using vertical bar charts with standard deviations.

For the first aim, the assumption of homogeneity of variance was tested using Levene statistics. To meet the need for non-parametric testing, Mann-Whitney $U$ tests were performed to compare the two groups.

For the second aim, the homogeneity of variance was also tested with Levene statistics and the three groups were

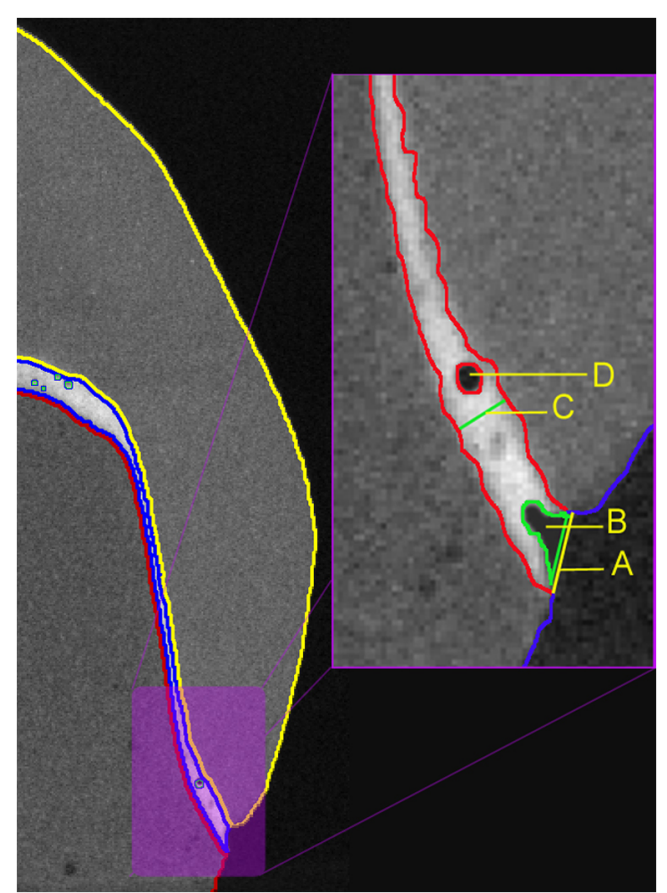

Fig. 3 2D representation of a cemented PICN material crown with the measuring parameters according to Holmes et al. [5]: (A) absolute marginal discrepancy; (B) porosities in the marginal area in contact with the external environment; (C) internal gap; (D) porosities inside the cement layer compared using one-way ANOVA followed by a post hoc Tukey test.

\section{Results}

\section{Influence of the cement space setting: $50 \mu \mathrm{m}$ versus $80 \mu \mathrm{m}$}

The micro-CT analysis and the measuring software made it possible to carry out 150 circumferential measurements of AMD on each crown and also to determine the mean IG for the whole cement space as well as the volumetric quantification of the TCV and VP.

Table 2 shows the mean values, minimum, maximum, and standard deviations of AMD, IG, TCV, and VP of the crowns manufactured with an internal gap of $50 \mu \mathrm{m}$ and $80 \mu \mathrm{m}$. The mean values for the $50 \mu \mathrm{m}$ group were the following: AMD $182.6 \mu \mathrm{m}$, IG $215.6 \mu \mathrm{m}$, TCV $22.9 \mathrm{~mm}^{3}$, and VP $0.26 \mathrm{~mm}^{3}$. The $80 \mu \mathrm{m}$ group showed the following mean values: AMD $211.9 \mu \mathrm{m}, \mathrm{IG} 173.1 \mu \mathrm{m}, \mathrm{TCV} 20.49 \mathrm{~mm}^{3}$, and VP $0.34 \mathrm{~mm}^{3}$. The statistical analysis revealed no significant difference in the measured parameters between both groups $(p>0.05)$.

\section{Influence of three different resin luting materials}

Table 3 shows the mean values, minimum, maximum, and standard deviations of AMD, IG, TCV, and VP of the crowns cemented with three different resin materials. The mean values of the RX group were the following: AMD $210.8 \mu \mathrm{m}, \mathrm{IG} 171.1 \mu \mathrm{m}, \mathrm{TCV} 17.57 \mathrm{~mm}^{3}$, and VP of $0.32 \mathrm{~mm}^{3}$. VLE group mean values were the following: AMD $195.5 \mu \mathrm{m}, \mathrm{IG} 198.6 \mu \mathrm{m}, \mathrm{TCV} 17.49 \mathrm{~mm}^{3}$, and VP $0.46 \mathrm{~mm}^{3}$. The NX3 group showed following values: AMD $186.6 \mu \mathrm{m}$, IG $203 \mu \mathrm{m}$, TCV $20.59 \mathrm{~mm}^{3}$, and VP of $0.54 \mathrm{~mm}^{3}$. The statistical analysis showed a significant difference between the RX and NX3 group in the volume of porosities in the marginal area $(p=0.033)$. No significant differences were found regarding the other parameters.

\section{Discussion}

The purpose of the present study was to investigate the influence of two different fitting parameters and three different resin luting materials in the marginal and internal fit of polymer-infiltrated ceramic network crowns manufactured after digital impressions with the $3 \mathrm{M}$ true definition scanner.

Based on the micro-CT measurements, the comparison of two different virtual cement space settings showed no statistically significant difference with regard to the parameters for accuracy of fit. The mean AMD values were $182.6 \mu \mathrm{m}$ for the $50 \mu \mathrm{m}$ group and $211.9 \mu \mathrm{m}$ for the $80 \mu \mathrm{m}$ group. These results 


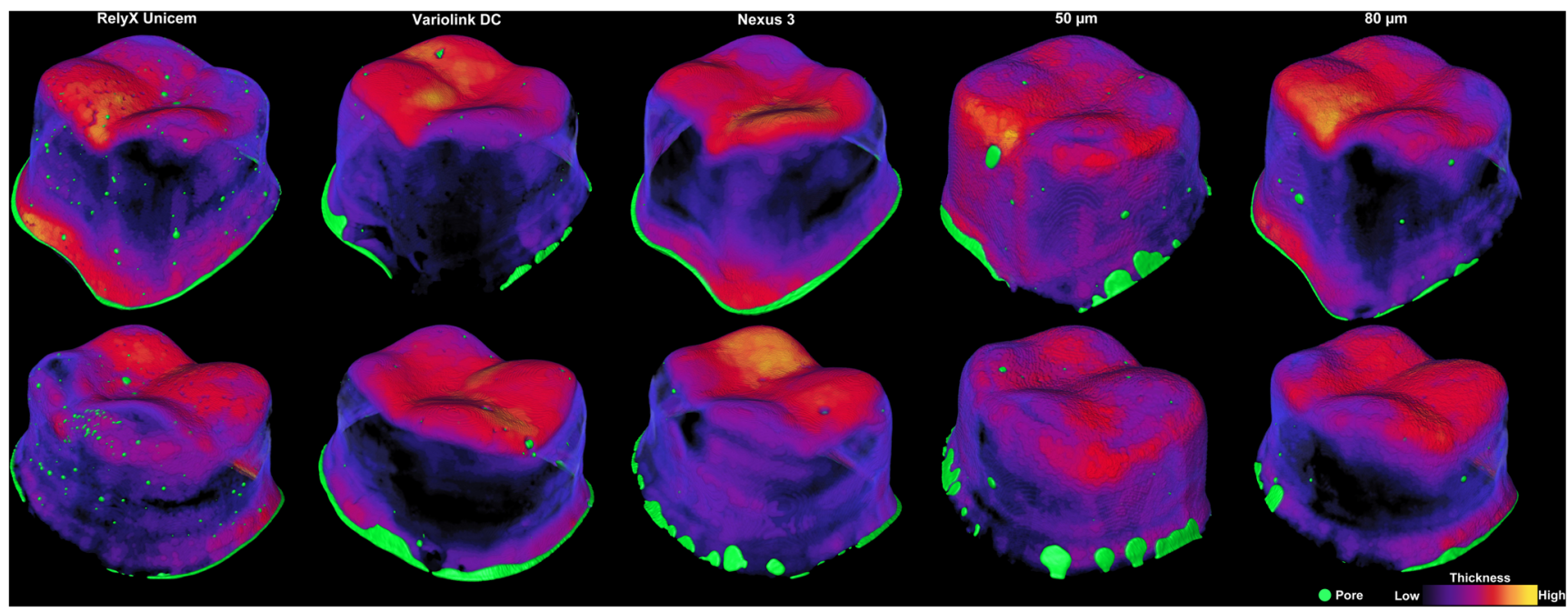

Fig. 4 Visualization of the cement space showing the cement thickness and porosities of representative specimens of each group. Thicker areas are represented with lighter color while thinner areas are represented with darker color. Porosities are shown with green color

indicate that an increase of the cement space to $80 \mu \mathrm{m}$ does not improve the marginal fit of the PICN crowns as proposed by Shim et al. [16]. Compared with the results of Yildirim et al., who measured a mean AMD of $102.4 \mu \mathrm{m}$ for Vita Enamic crowns, the AMD values of both groups in the present study are higher [15]. A possible explanation for these results might be that Yildirim et al. used another intra-oral scanner for the impression taking process and a different milling unit. A more plausible explanation could be that the crowns were not cemented but only stabilized on their respective dies with a silicone material as well as the fact that there was a different cement space width of $40 \mu \mathrm{m}[12,25]$. The mean AMD data of the $50 \mu \mathrm{m}$ group in the present study showed also higher values when compared with a previous investigation conducted by Dauti et al. [24]. However, all experiments of both studies were conducted in identical conditions using the same intra-oral scanner and same fitting parameters. Most likely, the reason for these discrepancies might be the influence of the operator, the dental technician, and the cementation procedure, which cannot be entirely standardized. Regarding the IG, the mean value for the $50 \mu \mathrm{m}$ group was $215.6 \mu \mathrm{m}$ and
$173.1 \mu \mathrm{m}$ for the $80 \mu \mathrm{m}$ group, respectively. An evenly distributed cement space is important for acceptable mechanical properties and good retention of the restoration [26, 27]. According to Liu et al., higher luting material thickness allows for a higher degree of flexure of the crown, thus causing larger tensile stress in the core of the crown. On the other hand, a thinner layer hinders the full absorption of mechanical energy by the luting material, thus also increasing the stress in the crown. These findings imply that the cement space thickness is of secondary importance compared with the influence of loading conditions or cement moduli [28]. The mean IG values of both groups do not reproduce the configured cement spaces in the design software, which is consistent with the results of previous research $[14,15,29]$. One explanation could be the preparation design, especially the convergence angle of the axial walls, which might affect the accuracy of fit. An increase in the convergence angle might possibly produce lower internal gap values [14]. The total volume of the cement space showed no significant difference between both groups, regardless of the space setting. Considering the fact that both groups had no significant difference between the measured

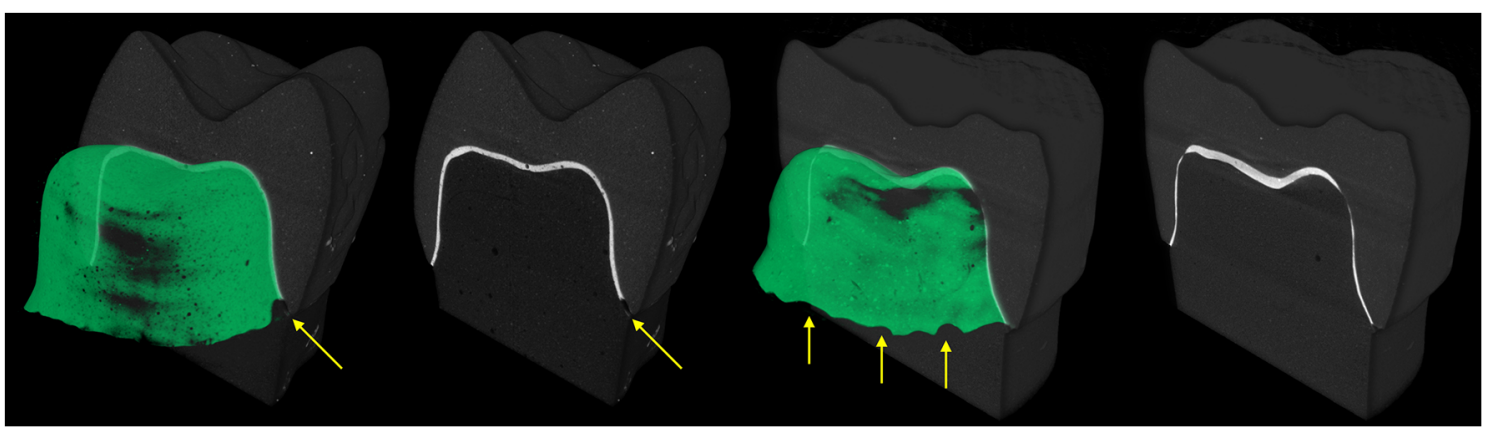

Fig. 5 Representative micro-CT images and visualization of two specimens showing the cement space and porosities in the marginal area with contact to the external environment. Volume rendering was performed using the software CT vox (Bruker micro-CT, Bruker Corporation, USA, version 3.3.0 r1403) 
Table 2 Values of the absolute marginal discrepancy, internal gap, total cement space volume, and volume of porosities in the marginal area for the $50 \mu \mathrm{m}$ and $80 \mu \mathrm{m}$ group

\begin{tabular}{llllll}
\hline Group & Parameters & Mean & SD & Min. & Max. \\
\hline $50 \mu \mathrm{m}$ & AMD & $182.6 \mu \mathrm{m}$ & $22.6 \mu \mathrm{m}$ & $120 \mu \mathrm{m}$ & $200 \mu \mathrm{m}$ \\
& IG & $215.6 \mu \mathrm{m}$ & $56.8 \mu \mathrm{m}$ & $140 \mu \mathrm{m}$ & $310 \mu \mathrm{m}$ \\
& TCV & $22.9 \mathrm{~mm}^{3}$ & $5.28 \mathrm{~mm}^{3}$ & $16.03 \mathrm{~mm}^{3}$ & $30.07 \mathrm{~mm}^{3}$ \\
& VP & $0.26 \mathrm{~mm}^{3}$ & $0.14 \mathrm{~mm}^{3}$ & $0.1 \mathrm{~mm}^{3}$ & $0.54 \mathrm{~mm}^{3}$ \\
$80 \mu \mathrm{m}$ & AMD & $211.9 \mu \mathrm{m}$ & $96.5 \mu \mathrm{m}$ & $120 \mu \mathrm{m}$ & $400 \mu \mathrm{m}$ \\
& IG & $173.1 \mu \mathrm{m}$ & $55.2 \mu \mathrm{m}$ & $140 \mu \mathrm{m}$ & $330 \mu \mathrm{m}$ \\
& TCV & $20.49 \mathrm{~mm}^{3}$ & $6.73 \mathrm{~mm}^{3}$ & $16.37 \mathrm{~mm}^{3}$ & $39.1 \mathrm{~mm}^{3}$ \\
& VP & $0.34 \mathrm{~mm}^{3}$ & $0.18 \mathrm{~mm}^{3}$ & $0.07 \mathrm{~mm}^{3}$ & $0.67 \mathrm{~mm}^{3}$ \\
\hline
\end{tabular}

marginal and internal fit parameters, for the second aim of the study, the cement space settings in the design software were set at $50 \mu \mathrm{m}$.

The second part of the investigation evaluated the influence of three different resin luting materials, RelyX Unicem (RX), Variolink Esthetic DC (VLE), and Nexus 3 (NX3), on the marginal and internal fit of cemented PICN material crowns. A significant difference between the groups was only found in the porosities in the cement space on the marginal area, which might negatively influence the long-term success of the restoration. The RX group showed the lowest mean volume of porosities. The other measured parameters showed no statistical difference. The mean AMD value for the RX group was $210.8 \mu \mathrm{m}$, for the VLE group $195.5 \mu \mathrm{m}$ and for the NX3 group $186.6 \mu \mathrm{m}$. The RX group resulted in approximately $30 \mu \mathrm{m}$ higher mean AMD compared with the aforementioned $50 \mu \mathrm{m}$ group and about $70 \mu \mathrm{m}$ higher AMD compared with a previous study by the authors, even though the manufacturing and cementation process took place under identical conditions [12]. The VLE group showed lower mean AMD values compared with a study conducted by Peroz et al. that investigated the marginal fit of cemented lithium disilicate ceramic crowns using 3 different luting materials, in which a mean AMD value of $412 \mu \mathrm{m}$ was measured for the Variolink group. On the other hand, the RelyX Unicem group showed a mean AMD of $213 \mu \mathrm{m}$, which is similar to the RX group of the present study [30]. For the NX3 group, no studies could be found to compare the marginal fit values. It is important to mention that Peroz et al. used different materials for the manufacture of the crowns compared with the present study. According to Azarbal et al., materials in which a crystallization firing is involved like, e.g., lithium disilicate (IPS e.max CAD) show poorer marginal adaptation compared with materials like Vita Enamic, which do not require this extra step [31]. IG mean values were $171.1 \mu \mathrm{m}$ for the RX group, $198.6 \mu \mathrm{m}$ for the VLE group, and $203 \mu \mathrm{m}$ for the NX3 group. Taken together, the AMD, IG, and TCV data of the cemented PICN crowns with 3 different resin luting materials showed variability between the groups, however, without a statistical significance between them. According to research, RelyX Unicem builds a film thickness of $15.1 \mu \mathrm{m} 1 \mathrm{~min}$ after mixing [32], while Variolink showed an initial film thickness between $18.4 \mu \mathrm{m}$ [33] and $22.2 \mu \mathrm{m}$ [34]. For Nexus 3, no data on film thickness were found. However, previous research on Nexus 2 detected a film thickness value of $18.6 \mu \mathrm{m}$ [34]. Correspondent with the ISO 9917 norm, these values are below the maximum specified value of $25 \mu \mathrm{m}$ for water-based cements [33], which theoretically should also apply to resin-based luting materials. It can therefore be assumed that the film thickness of the 3 tested materials should not affect the proper seating of the crowns. Nevertheless, all three groups had mean AMD values above the $120-\mu \mathrm{m}$ threshold for clinically acceptable margins [8]. In addition to factors like the impression technique and the manufacturing procedure, possible reasons for the higher values could have been the convergence angle of the axial walls, the seating pressure during cementation, and the flowing properties of the luting materials $[14,35]$. Lower
Table 3 Values of the absolute marginal discrepancy, internal gap, total cement space volume, and volume of porosities in the marginal area for the RelyX Unicem, Variolink Esthetic DC, and Nexus 3 group

\begin{tabular}{|c|c|c|c|c|c|}
\hline Group & Parameters & Mean & SD & Min. & Max. \\
\hline \multirow[t]{4}{*}{ RelyX } & AMD & $210.8 \mu \mathrm{m}$ & $55.2 \mu \mathrm{m}$ & $150 \mu \mathrm{m}$ & $330 \mu \mathrm{m}$ \\
\hline & IG & $171.1 \mu \mathrm{m}$ & $33.3 \mu \mathrm{m}$ & $120 \mu \mathrm{m}$ & $210 \mu \mathrm{m}$ \\
\hline & $\mathrm{TCV}$ & $17.57 \mathrm{~mm}^{3}$ & $3.41 \mathrm{~mm}^{3}$ & $12.28 \mathrm{~mm}^{3}$ & $23.42 \mathrm{~mm}^{3}$ \\
\hline & VP & $0.32 \mathrm{~mm}^{3}$ & $0.15 \mathrm{~mm}^{3}$ & $0.13 \mathrm{~mm}^{3}$ & $0.62 \mathrm{~mm}^{3}$ \\
\hline \multirow[t]{4}{*}{ Variolink } & AMD & $195.5 \mu \mathrm{m}$ & $25.7 \mu \mathrm{m}$ & $150 \mu \mathrm{m}$ & $240 \mu \mathrm{m}$ \\
\hline & IG & $198.6 \mu \mathrm{m}$ & $41.9 \mu \mathrm{m}$ & $130 \mu \mathrm{m}$ & $260 \mu \mathrm{m}$ \\
\hline & TCV & $17.49 \mathrm{~mm}^{3}$ & $3.07 \mathrm{~mm}^{3}$ & $13.49 \mathrm{~mm}^{3}$ & $21.63 \mathrm{~mm}^{3}$ \\
\hline & VP & $0.46 \mathrm{~mm}^{3}$ & $0.19 \mathrm{~mm}^{3}$ & $0.16 \mathrm{~mm}^{3}$ & $0.78 \mathrm{~mm}^{3}$ \\
\hline \multirow[t]{4}{*}{ Nexus 3} & AMD & $186.6 \mu \mathrm{m}$ & $28.4 \mu \mathrm{m}$ & $150 \mu \mathrm{m}$ & $240 \mu \mathrm{m}$ \\
\hline & IG & $203 \mu \mathrm{m}$ & $48.1 \mu \mathrm{m}$ & $130 \mu \mathrm{m}$ & $300 \mu \mathrm{m}$ \\
\hline & $\mathrm{TCV}$ & $20.59 \mathrm{~mm}^{3}$ & $4.32 \mathrm{~mm}^{3}$ & $14.27 \mathrm{~mm}^{3}$ & $28.49 \mathrm{~mm}^{3}$ \\
\hline & VP & $0.54 \mathrm{~mm}^{3}$ & $0.19 \mathrm{~mm}^{3}$ & $0.32 \mathrm{~mm}^{3}$ & $0.91 \mathrm{~mm}^{3}$ \\
\hline
\end{tabular}


AMD values might have been achieved if dynamic loading during the seating of the crowns had been performed $[35,36]$.

The manufacture of the crowns in this study was done strictly digitally, including the impression procedure, the virtual design, and the milling of the crowns. This process, compared with the conventional manufacture of restorations, eliminates some working steps; however, it introduces new variables, e.g., software versions of the intra-oral scanner and the designing software which could influence the overall adaptation of the restoration. Shim et al. examined the influence of different software versions of the intra-oral scanner and design software of a CEREC system on the accuracy of crowns milled from resin nano-ceramic blocks, concluding that later software versions produce more accurate restorations [16]. Consequently, the results of this investigation apply for the devices that are used in the study with the software versions as stated above and might be different from newer versions. The material used for the fabrication of the dies was resinbased and clearly could not mimic a prepared tooth. Nevertheless, we decided to treat the die surface as if it was dentine in order to better simulate the clinical situation. In this case, the primer used in the VLE and NX3 group does not generate a hybrid layer with the die surface, which according to Loguercio et al. can compensate the stress that occurs during polymerization [37]. Cautiously interpreted, the stress during polymerization can lead to debonding and subsequently to an increase of the marginal discrepancies and porosities. Adjustments on the inner surface of the crowns before cementation to eliminate possible premature contacts between the crown and the die were not performed, in order to avoid biasing of the marginal values [38]. However, premature contacts could have also influenced the proper seating of the crown and subsequently increase the marginal discrepancies. The evaluation of accuracy fit of restorations by micro-CT gives a more detailed view of the relation between the restoration, luting agent, and the die. Considering this fact, it is difficult to interpret and compare the results with previous studies because of variations in the number of measurements per specimen, variety of the measuring points, and the sample size [9]. In the present study, the AMD was measured in 150 equidistant points circumferentially on the crown margin. Moreover, the IG was calculated as a mean value for the whole cement space. Consequently, with the high number of measurements, many of the discrepancies on the margin and the internal space are detected. These can subsequently lead to higher values of AMD and IG.

Porosities in the cement space on the marginal area with contact to the external environment were detected in all investigations. This fact is of clinical importance, since fluids, bacteria, and bacterial toxins can penetrate these spaces and get access to the unprotected dentin, compromising the success of the restoration [39]. When comparing the $50 \mu \mathrm{m}$ and $80 \mu \mathrm{m}$ group, no statistical difference between the groups was found.
The mean VP value of the $50 \mu \mathrm{m}$ group was $0.26 \mathrm{~mm}^{3}$, which is consistent with a previous study by the authors [24]. On the other hand, the $80 \mu \mathrm{m}$ group had a slightly elevated mean VP of $0.34 \mathrm{~mm}^{3}$. It seems that an increase in the cement gap to $80 \mu \mathrm{m}$ does not significantly affect the VP. However, a statistically significant difference was noticed between the RX and NX3 group. The mean VP volume of the RX group was $0.32 \mathrm{~mm}^{3}$, which is in agreement with the previous research by the authors [24]. NX3 had a mean VP volume of $0.54 \mathrm{~mm}^{3}$ and VLE a mean VP volume of $0.46 \mathrm{~mm}^{3}$. Research reveals that factors such as differences in particle size and mixing technique might contribute to the formation of porosities $[19,40]$. RelyX Unicem has an approximate particle size of $12.5 \mu \mathrm{m}$ compared with Nexus 3 with a particle size of $0.6 \mu \mathrm{m}$ and Variolink Esthetic with a particle size of $0.1 \mu \mathrm{m}$. Cautiously interpreting these findings, we assume that the resin material with the larger particle size produced less porosities in the marginal area. The mixing procedure for all three materials was performed according to the manufacturer's instructions. In accordance with previous research, hand mixing of luting agents leads to more air inclusion compared with automatic mixing [41]. Unlike VLE and NX3, which were mixed in the automix syringe, the RX was mixed in a Rotomix capsule mixing unit. The Rotomix mixing unit could be in this case more effective in removing porosities inside the resin material due to its rotational and centrifugal action causing the large pores to break during the initial mixing process [42]. In whichever way and however they occurred, porosities formed in the cement space can be considered destructive not only because of the penetration of fluids and bacteria but also mechanically, as it allows deformation of the material under loading conditions [40].

This study has limitations due to the in vitro setting of the experiments. Clinical factors including patients' movements during the impression procedure, subgingival preparation margins, or contaminations with blood or saliva cannot be taken into consideration. Also, the digitization of the dies without the adjacent teeth could have influenced the accuracy of the impression. Furthermore, the cementation procedure by firstly using finger pressure by the operator can influence the correct placement of the crown and might lead to larger discrepancies on a particular site. In addition, PICN material, luting agent, and the resin show similar X-ray absorption coefficients whereby the correct recognition of the borders between these materials is affected, resulting in either wider or thinner gaps. On the other hand, with this study, we were able to test the accuracy of the whole digital manufacturing process of PICN crowns including the cementation procedure using high-resolution micro-CT imaging combined with modern measuring and visualizing software. Furthermore, we accomplished to detect and visualize porosities in each of the resin materials used and quantify those appearing in the marginal area. Further research should be conducted on testing of the 
latest $\mathrm{CAD} / \mathrm{CAM}$ workflows and on methods minimizing the appearance of porosities in the cement space.

\section{Conclusions}

In summary, within the limits of this study, we can conclude that different virtual spacer settings of $50 \mu \mathrm{m}$ and $80 \mu \mathrm{m}$ when Ceramill Mind (software version 3.4.10.1163) is used had no significant influence on the marginal and internal fit of cemented PICN material crowns. Additionally, no significant difference was found on the marginal and internal fit when PICN material crowns were cemented using three different resin-based materials. Porosities in the marginal area were found in all five groups, however, with significantly less porosities when using RelyX Unicem compared with Nexus 3. Since porosities in the cement space present a negative factor in the long-term survival of restorations, further research should be undertaken with focus on minimizing porosities.

Acknowledgments The authors would like to thank the following persons, institutions, and companies for their support: Jan Moestl, Fidelis Baur, Blerti Dauti, Dental Lab - Dental School Vienna, Institute of Anthropology - Vienna, 3M ESPE, and Amann Girrbach.

Funding Information Open access funding provided by Medical University of Vienna.

\section{Compliance with ethical standards}

Conflict of interest The authors declare that they have no conflict of interest.

Ethical approval This article does not contain any studies with human participants or animals performed by any of the authors.

Informed consent For this type of study, formal consent is not required.

Open Access This article is distributed under the terms of the Creative Commons Attribution 4.0 International License (http:// creativecommons.org/licenses/by/4.0/), which permits unrestricted use, distribution, and reproduction in any medium, provided you give appropriate credit to the original author(s) and the source, provide a link to the Creative Commons license, and indicate if changes were made.

\section{References}

1. Zimmermann M, Mehl A, Reich S (2013) New CAD/CAM materials and blocks for chairside procedures. Int J Comput Dent 16(2): 173-181

2. Kurbad A, Kurbad S (2013) A new, hybrid material for minimally invasive restorations in clinical use. Int J Comput Dent 16(1):69-79

3. Mainjot AK, Dupont NM, Oudkerk JC, Dewael TY, Sadoun MJ (2016) From artisanal to CAD-CAM blocks: state of the art of indirect composites. J Dent Res 95(5):487-495. https://doi.org/10. $1177 / 0022034516634286$
4. Furtado de Mendonca A, Shahmoradi M, Gouvea CVD, De Souza GM, Ellakwa A (2019) Microstructural and mechanical characterization of CAD/CAM materials for monolithic dental restorations. J Prosthodont 28(2):e587-e594. https://doi.org/10.1111/jopr.12964

5. Awada A, Nathanson D (2015) Mechanical properties of resinceramic CAD/CAM restorative materials. J Prosthet Dent 114(4): 587-593. https://doi.org/10.1016/j.prosdent.2015.04.016

6. Boitelle P, Mawussi B, Tapie L, Fromentin O (2014) A systematic review of $\mathrm{CAD} / \mathrm{CAM}$ fit restoration evaluations. J Oral Rehabil 41(11):853-874. https://doi.org/10.1111/joor.12205

7. Holmes JR, Bayne SC, Holland GA, Sulik WD (1989) Considerations in measurement of marginal fit. J Prosthet Dent 62(4):405-408

8. McLean JW, Fraunhofer JA (1971) The estimation of cement film thickness by an in vivo technique. Br Dent J 131(3):107-111

9. Contrepois M, Soenen A, Bartala M, Laviole O (2013) Marginal adaptation of ceramic crowns: a systematic review. J Prosthet Dent 110(6):447-454.e410. https://doi.org/10.1016/j.prosdent.2013.08.003

10. Kim J-H, Jeong J-H, Lee J-H, Cho H-W (2016) Fit of lithium disilicate crowns fabricated from conventional and digital impressions assessed with micro-CT. J Prosthet Dent 116(4):551-557. https://doi.org/10.1016/j.prosdent.2016.03.028

11. Kale E, Seker E, Yilmaz B, Ozcelik TB (2016) Effect of cement space on the marginal fit of CAD-CAM-fabricated monolithic zirconia crowns. J Prosthet Dent 116(6):890-895. https://doi.org/10. 1016/j.prosdent.2016.05.006

12. Borges GA, Faria JS, Agarwal P, Spohr AM, Correr-Sobrinho L, Miranzi BAS (2012) In vitro marginal fit of three all-ceramic crown systems before and after cementation. Oper Dent 37(6):641-649. https://doi.org/10.2341/11-012-1

13. Wang CJ, Millstein PL, Nathanson D (1992) Effects of cement, cement space, marginal design, seating aid materials, and seating force on crown cementation. J Prosthet Dent 67(6):786-790

14. Iwai T, Komine F, Kobayashi K, Saito A, Matsumura H (2008) Influence of convergence angle and cement space on adaptation of zirconium dioxide ceramic copings. Acta Odontol Scand 66(4): 214-218. https://doi.org/10.1080/00016350802139833

15. Yildirim G, Uzun IH, Keles A (2017) Evaluation of marginal and internal adaptation of hybrid and nanoceramic systems with microcomputed tomography: an in vitro study. J Prosthet Dent 118(2):200-207. https://doi.org/10.1016/j.prosdent.2016.11.005

16. Shim JS, Lee JS, Lee JY, Choi YJ, Shin SW, Ryu JJ (2015) Effect of software version and parameter settings on the marginal and internal adaptation of crowns fabricated with the CAD/CAM system. J Appl Oral Sci 23(5):515-522. https://doi.org/10.1590/1678775720150081

17. Peumans M, Valjakova EB, De Munck J, Mishevska CB, Van Meerbeek B (2016) Bonding effectiveness of luting composites to different CAD/CAM materials. J Adhes Dent 18(4):289-302. https://doi.org/10.3290/j.jad.a36155

18. Edelhoff D, Ozcan M (2007) To what extent does the longevity of fixed dental prostheses depend on the function of the cement? Working group 4 materials: cementation. Clin Oral Implants Res 18(Suppl 3):193-204. https://doi.org/10.1111/j.1600-0501.2007.01442.x

19. Malkoç MA, Sevimay M, Tatar İ, Çelik HH (2015) Micro-CT detection and characterization of porosity in luting cements. J Prosthodontics 24:553-561. https://doi.org/10.1111/jopr.12251

20. Goracci C, Cury AH, Cantoro A, Papacchini F, Tay FR, Ferrari M (2006) Microtensile bond strength and interfacial properties of selfetching and self-adhesive resin cements used to lute composite onlays under different seating forces. J Adhes Dent 8(5):327-335

21. Doube M, Klosowski MM, Arganda-Carreras I, Cordelieres FP, Dougherty RP, Jackson JS, Schmid B, Hutchinson JR, Shefelbine SJ (2010) BoneJ: free and extensible bone image analysis in ImageJ. Bone 47(6):1076-1079. https://doi.org/10.1016/j.bone. 2010.08.023 
22. Schneider CA, Rasband WS, Eliceiri KW (2012) NIH Image to ImageJ: 25 years of image analysis. Nat Methods 9(7):671-675

23. Schindelin J, Arganda-Carreras I, Frise E, Kaynig V, Longair M, Pietzsch T, Preibisch S, Rueden C, Saalfeld S, Schmid B, Tinevez JY, White DJ, Hartenstein V, Eliceiri K, Tomancak P, Cardona A (2012) Fiji: an open-source platform for biological-image analysis. Nat Methods 9(7):676-682. https://doi.org/10.1038/nmeth.2019

24. Dauti R, Cvikl B, Lilaj B, Heimel P, Moritz A, Schedle A (2019) Micro-CT evaluation of marginal and internal fit of cemented polymer infiltrated ceramic network material crowns manufactured after conventional and digital impressions. J Prosthodont Res 63(1):40 46. https://doi.org/10.1016/j.jpor.2018.04.005

25. Kale E, Yilmaz B, Seker E, Özcelik TB (2017) Effect of fabrication stages and cementation on the marginal fit of CAD-CAM monolithic zirconia crowns. J Prosthet Dent 118:736-741. https://doi.org/ 10.1016/j.prosdent.2017.01.004

26. de Paula Silveira AC, Chaves SB, Hilgert LA, Ribeiro APD (2017) Marginal and internal fit of CAD-CAM-fabricated composite resin and ceramic crowns scanned by 2 intraoral cameras. J Prosthet Dent 117(3):386-392. https://doi.org/10.1016/j.prosdent.2016.07.017

27. Kim JH, Miranda P, Kim DK, Lawn BR (2003) Effect of an adhesive interlayer on the fracture of a brittle coating on a supporting substrate. J Mater Res 18(01):222-227. https://doi.org/10.1557/jmr.2003.0031

28. Liu B, Lu C, Wu Y, Zhang X, Arola D, Zhang D (2011) The effects of adhesive type and thickness on stress distribution in molars restored with all-ceramic crowns. J Prosthodontics 20(1):35-44. https://doi.org/10.1111/j.1532-849X.2010.00650.x

29. Prudente MS, Davi LR, Nabbout KO, Prado CJ, Pereira LM, Zancopé K, Neves FD (2017) Influence of scanner, powder application, and adjustments on CAD-CAM crown misfit. J Prosthet Dent 119:377-383. https://doi.org/10.1016/j.prosdent.2017.03.024

30. Peroz I, Mitsas T, Erdelt K, Kopsahilis N (2018) Marginal adaptation of lithium disilicate ceramic crowns cemented with three different resin cements. Clin Oral Investig 23:315-320. https://doi.org/ 10.1007/s00784-018-2438-6

31. Azarbal A, Azarbal M, Engelmeier RL, Kunkel TC (2017) Marginal fit comparison of CAD/CAM crowns milled from two different materials. J Prosthodontics 27:421-428. https://doi.org/ 10.1111/jopr. 12683
32. Kious AR, Roberts HW, Brackett WW (2009) Film thicknesses of recently introduced luting cements. J Prosthet Dent 101(3):189192. https://doi.org/10.1016/s0022-3913(09)60026-3

33. Osman SA, McCabe JF, Walls AWG (2006) Film thickness and rheological properties of luting agents for crown cementation. Eur J Prosthodont Restor Dent 14(1):23-27

34. Bagheri R (2013) Film thickness and flow properties of resin-based cements at different temperatures. J Dentistry (Shiraz, Iran) 14(2): $57-63$

35. Tsukada G, Tanaka T, Kajihara T, Torii M, Inoue K (2006) Film thickness and fluidity of various luting cements determined using a trial indentation meter. Dent Mater 22(2):183-188. https://doi.org/ 10.1016/j.dental.2005.04.014

36. Rosenstiel SF, Land MF, Crispin BJ (1998) Dental luting agents: a review of the current literature. J Prosthet Dent 80(3):280-301

37. Loguercio AD, Reis A, Bortoli G, Patzlaft R, Kenshima S, Rodrigues Filho LE, Accorinte Mde L, van Dijken JW (2006) Influence of adhesive systems on interfacial dentin gap formation in vitro. Oper Dent 31(4):431-441. https://doi.org/10.2341/05-53

38. Boening KW, Wolf BH, Schmidt AE, Kästner K, Walter MH (2000) Clinical fit of Procera AllCeram crowns. J Prosthet Dent 84(4):419-424. https://doi.org/10.1067/mpr.2000.109125

39. Milutinović-Nikolić AD, Medić VB, Vuković ZM (2007) Porosity of different dental luting cements. Dent Mater 23(6):674-678. https://doi.org/10.1016/j.dental.2006.06.006

40. Ciucchi B, Bouillaguet S, Delaloye M, Holz J (1997) Volume of the internal gap formed under composite restorations in vitro. J Dent 25(3-4):305-312. https://doi.org/10.1016/s0300-5712(96)00032-2

41. Fleming GJP, Zala DM (2003) An assessment of encapsulated versus hand-mixed glass ionomer restoratives. Oper Dent 28(2):168-177

42. Fleming GJP, Kenny SM, Barralet JE (2006) The optimisation of the initial viscosity of an encapsulated glass-ionomer restorative following different mechanical mixing regimes. J Dent 34(2): 155-163. https://doi.org/10.1016/j.jdent.2005.05.008

Publisher's note Springer Nature remains neutral with regard to jurisdictional claims in published maps and institutional affiliations. 